\title{
Robot@Factory Lite Competition: A Digital Twin Approach for the AGV
}

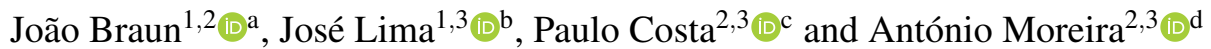 \\ ${ }^{1}$ Research Centre of Digitalization and Intelligent Robotics, Polytechnic Institute of Bragança, Campus de Santa Apolónia, \\ Bragança, Portugal \\ ${ }^{2}$ Faculty of Engineering of University of Porto (FEUP), University of Porto, R. Dr. Roberto Frias, Porto, Portugal \\ ${ }^{3}$ INESC Technology and Science, FEUP, R. Dr. Roberto Frias, Porto, Portugal \\ jllima\}@ipb.pt, amoreira\}@fe.up.pt
}

Keywords: Digital Twin, Robotics Competitions, AGV, Simulation, DC Motors.

Abstract: Robotics competitions are environments that foster teamwork, AI, and technology development by encouraging students, researchers, and academics to test their solutions against each other. These competitions often challenge the competitors' prototypes with tasks specifically designed to benchmark them with the current optimal solutions. During the prototype stages of a robot, the development costs and time spent are often higher than other stages, as changes in the prototype are frequent. Simulation is often used to reduce these variables as it allows flexibility in all development stages before transitioning to the real scenario. However, a digital twin can be used to increase even further flexibility and effectiveness. Digital twins are virtual representations of real assets, providing replication and prediction of real scenario events, and real-time monitoring of the real object. Thus, this paper presents the development of a digital twin of an automatic guided vehicle (AGV) to the Robot@ Factory Lite competition and the tests performed to validate the approach.

\section{INTRODUCTION}

Robotics competitions are one of the approaches that drive technology development as it encourages researchers, academics, and students to develop solutions to the tasks offered by the competitions. They are known to challenge state-of-the-art technologies and their algorithms' performance, to later be adopted in the industry.

Having Robot@Factory competition as a base, that started in 2011 Portuguese Robotics Open, the Robot@Factory Lite (R@FL) is a simplified version where the parts should be transported and organized between warehouses and processing machines through a magnet, bringing this competition more accessible for younger students. For further information, the reader is referred to (Lima et al., 2019). The organization provides a prototype and some libraries to deal with the robot's I/O (magnet, RFID, motors, etc.) to facilitate the development for new participants. Moreover, it is provided a simulation scene

\footnotetext{
a (iD) https://orcid.org/0000-0003-0276-4314

b (iD) https://orcid.org/0000-0001-7902-1207

c (iD https://orcid.org/0000-0002-4846-271X

d(D) https://orcid.org/0000-0001-8573-3147
}

from the Simtwo simulator where a hardware-in-theloop was developed.

During the development of a robot as a whole, prototyping stages costs and time spent are usually higher than the others. This happens by the fact that these stages are constituted by frequent hardware and software changes. Whenever a new change is made and needs to be tested, it takes time to test in the real robot. Not to mention that the test location can be a different place from where the workplace is. Thus, simulations are used to mitigate these inconveniences by increasing flexibility during these stages, provided they simulate realistically the real robot. Even so, frequently the transition to the real scenario needs adjustments, as realistically simulations are computationally expensive.

To increase even further the effectiveness and flexibility of the prototype stages of the robot for the R@FL competition, a digital twin was developed. In this case, the digital twin is a realistic model of the real robot that will run simultaneously with it. The twins communicate in real-time, providing monitoring data throughout a set of embedded sensors on the real robot. Thus, the development of the robot's approach to the competition will benefit from instant feedback of the algorithms. Moreover, by conse- 
quence, the transition to the real scenario will be greatly improved, if not outright removed, as the simulation will operate similarly to the real robot.

The paper is organized as follows: after this introduction, the related work is presented in Section 2. Section 3 briefly describes the entire system's setup. Section 4 addresses the methodology. The performed tests and their respective results are highlighted in Section 5. The conclusions with future works are stressed in Sections 6.

\section{STATE OF THE ART}

Digital twins are a broad concept that is applied in several different areas of industry. The industrial applications focus on the areas of design, production, prognostic and health management, among others (Tao et al., 2019). In this way, a few examples of these areas are presented below.

In product design, a digital twin-based approach for designing hollow glass production lines was presented in (Zhang et al., 2017). The authors validated their approach using a case study in hollow glass production line.

Regarding the production area, a framework for modeling and simulation of Cyber-Physical Production Systems is presented in (Weyer et al., 2016). According to the authors, the framework was currently being implemented and validated within the automotive industry.

According to (Tao et al., 2019), most of the applications of Digital Twins are applied to prognostics and health management (PHM). A digital twin approach was proposed for complex equipment (Tao et al., 2018). The authors used a case study of gearbox prognosis to confirm their approach for improving the accuracy of prognosis.

Finally, a simulated twin for a remote energy platform was presented in (Pairet et al., 2019). According to the authors, the ORCA Hub Simulator is an oil rig environment that includes three types of autonomous systems (Husky, ANYmal, and UAVs) for human-robot collaboration scenarios.

\section{THE SETUP}

The assembled robot follows the schematic recommended by the competition (P33a, 2020), and can be seen in the figure below.

The robot has a differential drive configuration. It has two DFRobot TT Micro DC geared motors with

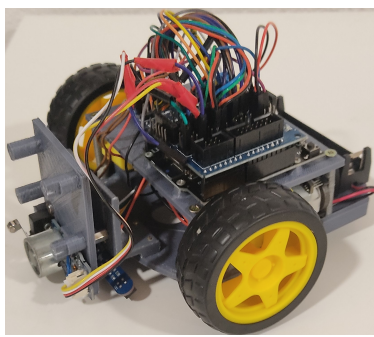

Figure 1: Real robot.

integrated quadrature encoders and one caster wheel for stability. The encoders provide 16 pulses per rotation per channel. Together with the 120:1 gearbox, the resolution reaches 3840 ticks per revolution.

The digital twin system will work as follows:

- The real robot will operate normally.

- The simulated robot will communicate with an ESP8266 by serial communication to perform the hardware in the loop.

- Both of them will communicate, by UDP protocol, with monitoring software, the digital twin software.

The entire system can be seen in Figure 2.

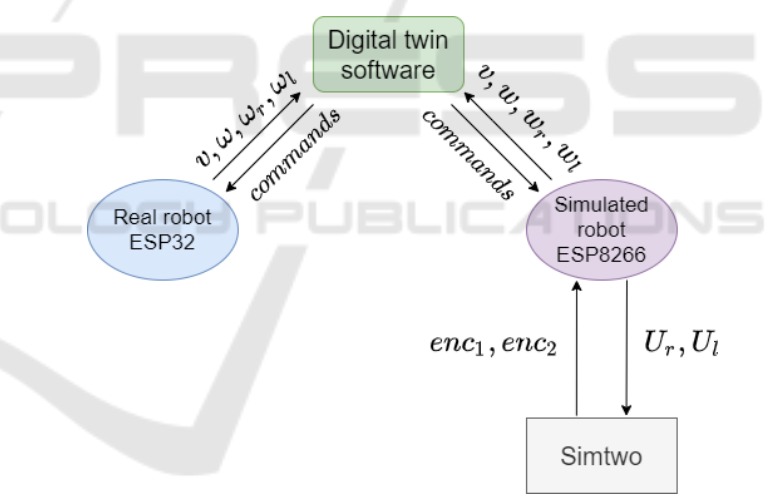

Figure 2: Digital twin system's diagram.

Where $v$ and $\omega$ are the robot's linear and angular speeds respectively. The right and left motors' angular speeds are denoted by $\omega_{r}$ and $\omega_{l}$. The encoder readings are represented by $e n c_{1}$ and $e n c_{2}$. Finally, the right and left motors' voltages are displayed by $U_{r}$ and $U_{l}$.

\section{METHODOLOGY}

SimTwo simulator was chosen for this project for both the organization providing a simulation scene for the competition as well as being a realistic 3D simulator with rigid body interactions and constraints (Paulo et al., 2011). Regarding the physics simula- 
tion, SimTwo uses the Open Dynamics Engine (ODE) to perform the computations (Smith et al., 2005).

To construct the simulated robot model, all the robot's parts were measured and weighted. The model can be seen in Figure 3.

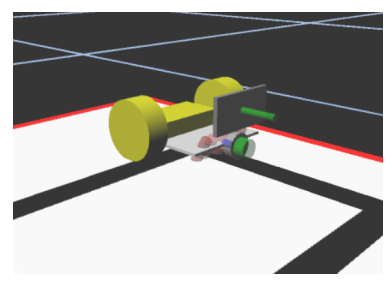

Figure 3: Simulated robot.

\subsection{Motors Models}

To simulate the motor models, first, it is necessary to obtain the DC motor parameters. Thus, steady-state and transient tests were performed for each motor.

\subsubsection{Steady State Tests}

The steady-state tests were performed with the robot in the air so that the motors' parameters were not influenced by the robot's weight. Before performing the tests, it is necessary to derive the motor models. In this way, the electric model of the armature of a generic DC motor can be seen in Figure 4.

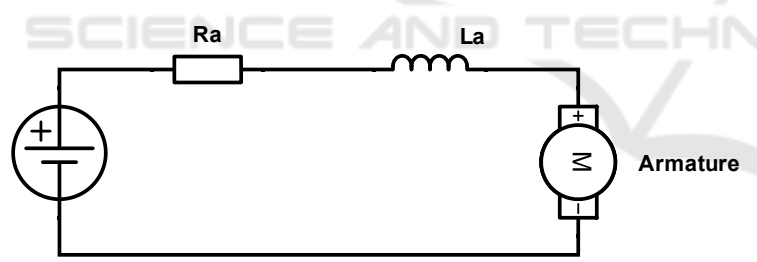

Figure 4: Electrical equivalent circuit of a DC motor's armature.

Applying Kirchoff Voltage Law to the circuit we have:

$$
U=R_{a} \cdot i_{a}+L \cdot \frac{d i_{a}}{d t}+e_{v}
$$

The inductance was assumed zero to simplify the calculations. This assumption is reasonable as the motors are small, and the equivalent armature inductance is, in general, very small for small motors. The voltage drop across the armature is $e_{v}$, the back emf generated by the motor while doing work, and it is proportional to the angular speed of the shaft. The proportionality constant is $k$, called the back emf constant.

$$
e_{v}=k \cdot \omega
$$

Substituting equation 2 in equation 1 and dividing it by $\omega$ we have:

$$
\frac{U}{\omega}=R_{a} \cdot \frac{i_{a}}{\omega}+k
$$

Now, with equation 3 , it is possible to perform a series of steady-state tests where a known voltage is applied to the motor, and its respective armature current and the axle angular speed are measured. The voltages were measured by a voltmeter and the currents by an ammeter. The angular speeds were estimated using the embedded encoders on the motors. In total, fifteen tests were made. The voltages ranged from $[0-6 V]$, which the upper limit is the nominal voltage for these motors. After gathering the data, a linear model was fit to obtain the angular coefficient $R_{a}$ and the linear coefficient $k$. Figures 5 and 6 show the results from the tests performed on each motor.

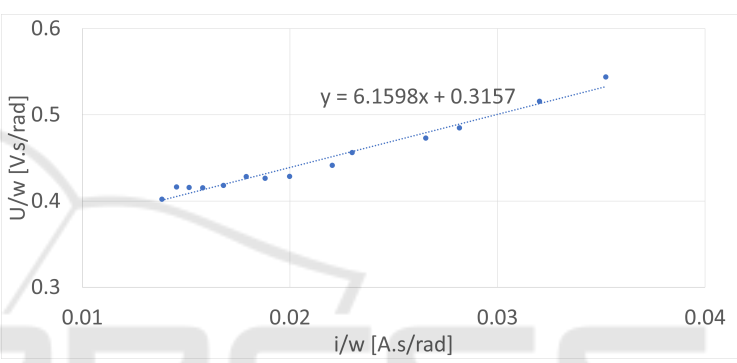

Figure 5: Linear model fit to the right motor data derived from tests applied to equation 3 .

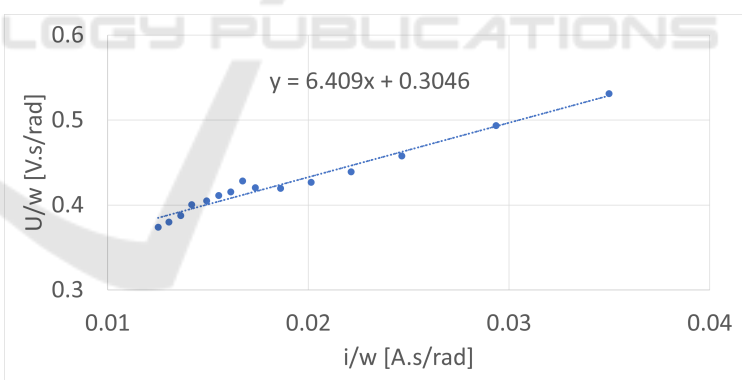

Figure 6: Linear model fit to the left motor data derived from tests applied to equation 3 .

The armature resistance and the back emf constant from each motor can be seen in Table 1 .

Applying the rotational variation of Newton's second law to the motor we have:

$$
T_{m}=T_{q}+B \cdot \omega+J \cdot \dot{\omega}
$$

Assuming steady-state, $\dot{\omega}$ is zero. The torque static friction is $T_{q}$ and $\mathrm{B}$ is the viscous friction constant. The motor torque is $T_{m}$ and it is proportional to the armature current, $T_{m}=k * i_{a}$. Substituting this equation into equation 5 , and rearranging the factors we have:

$$
k \cdot i_{a}=B \cdot \omega+T_{q}
$$


Following the same reasoning as the previous tests, fifteen tests were performed for each motor and a linear model was fit for each motors' data. The right and left motor tests can be seen in Figures 7 and 8, respectively.

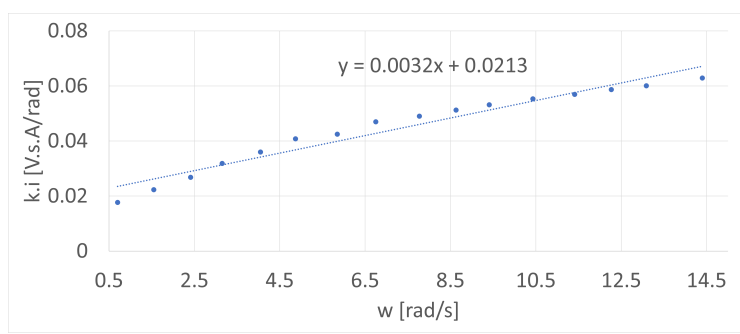

Figure 7: Linear model fit to the right motor data derived from tests applied to equation 5 .

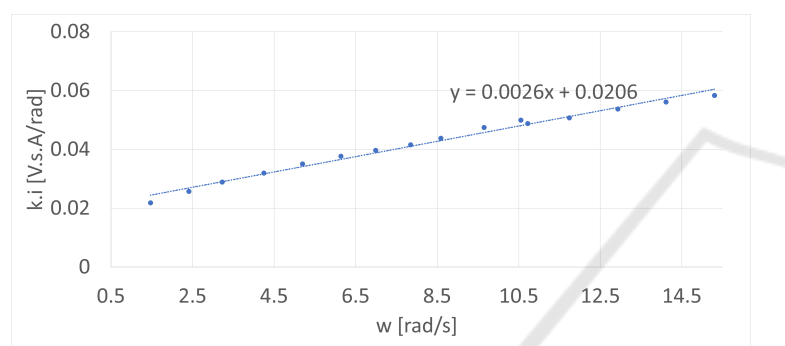

Figure 8: Linear model fit to the left motor data derived from tests applied to equation 5 .

Again, from these tests, the angular coefficient corresponds to the viscous friction and the linear coefficient to the torque static friction. The values of these parameters for each motor can be seen in Table 1 .

\subsubsection{Transient Tests}

To obtain the inertia of the motors it is necessary to perform transient tests. They were performed with the robot suspended on the air so that the resulting moment of inertia derived from the tests takes only into account the contribution of the rotor plus the wheel for each motor. The transfer function of the angular speed of a DC motor in relation to the input voltage can be seen in equation 6 .

$$
H(s)=\frac{\omega(s)}{U(s)}=\frac{\frac{k}{B R_{a}+k^{2}}}{\frac{J R_{a}}{B R_{a}+k^{2}} s+1}
$$

The constants are the DC motors parameters. Again, it was assumed that the inductance was zero for simplicity reasons. As it can be seen, this is a first-order system where the time constant factor is represented by $\frac{J R_{a}}{B R_{a}+k^{2}}$. To obtain $J$, a voltage step was applied to the motors and their time constants, $\tau$, were measured. With them, the only unknown constant is the moment of inertia. Thus, the time constant factor was solved for $J$ for each motor. All the obtained parameters, for each motor, can be seen in Table 1 .

Table 1: Parameters of each DC motor.

\begin{tabular}{|c|c|c|}
\hline Parameters & Right motor & Left motor \\
\hline \hline $\mathrm{Ra}[\Omega]$ & 6.16 & 6.41 \\
\hline $\mathrm{k}\left[\frac{\mathrm{V.s}}{\mathrm{rad}}\right]$ & 0.32 & 0.30 \\
\hline $\mathrm{Tq}[\mathrm{N} . \mathrm{m}]$ & 0.021 & 0.021 \\
\hline $\mathrm{B}\left[\frac{\mathrm{N.m.S}}{\mathrm{rad}}\right]$ & $3.2 \mathrm{E}-03$ & $2.6 \mathrm{E}-03$ \\
\hline $\mathrm{J}\left[\frac{\mathrm{N.m.s}}{\mathrm{rad}}\right]$ & $3.39 \mathrm{E}-03$ & $3.42 \mathrm{E}-03$ \\
\hline$\tau[\mathrm{s}]$ & 0.175 & 0.2 \\
\hline
\end{tabular}

All these parameters, together with all the robot's parts dimensions and weights, were applied to the simulation model in Simtwo. To measure the weights, a precision scale was used. As for the dimensions, a pachymeter. After that, transient tests were performed on the simulated robot's motors to see their behaviors in respect to the same step responses applied to the real robot. The tests applied to both robots can be seen in Figure 9.

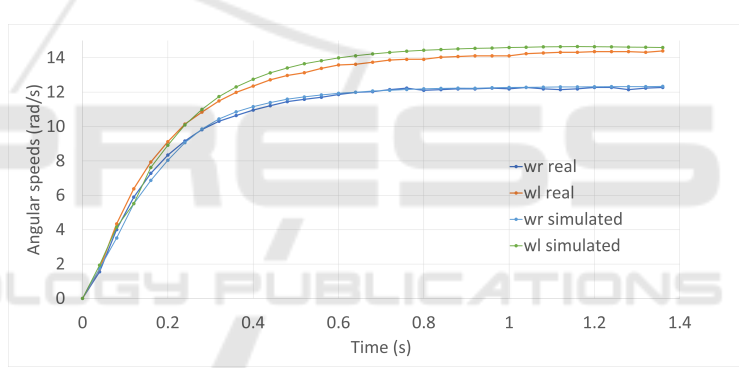

Figure 9: Right and left motors' open loop step responses from both robots.

It is clear to see from these responses that the real robot's motors have similar responses compared to their respective simulated ones. Also, it is possible to see that the real motors' responses are different from each other despite being applied the same step input and being the same model. The internal structure of the motors can influence this behavior. Although the simulated motors behave similarly in respect of their respective real motors, small differences are present. These outcomes can be influenced by a series of factors such as:

- Intrinsic errors in the measurements caused by the measurement devices (voltages, currents, angular speeds, weights, dimensions).

- Added errors by approximation.

- Inserted errors by linear regression estimation.

- Sampling period is limited to approximately 40 ms of the execution period. 


\subsection{PI Controller}

Simple PI controllers for angular speed were designed for each motor of the real robot. To design the controllers, the Internal Model Control (IMC) tuning method was applied (Rivera et al., 1986). As our model is a first-order system, the gains were computed in the way is displayed in Table 2.

Table 2: PI controller for first order transfer function with IMC tuning method.

\begin{tabular}{|c|c|}
\hline & PI control \\
\hline Kc & $\frac{\tau}{g_{p} \cdot\left[\tau_{c l}+t_{d}\right]}$ \\
\hline$T_{i}$ & $\tau$ \\
\hline$T_{d}$ & 0 \\
\hline
\end{tabular}

Where $\tau$ is the open-loop response time, $g_{p}$ is the system's open-loop gain, $\tau_{c l}$ is the desired closed-loop response time and, $t_{d}$, the dead time.

The PI controllers, for both motors of the real robot, were designed for a closed-loop time response of $200 \mathrm{~ms}$, which is a similar value to the highest openloop time response of both motors. Deadtime was considered zero. The values needed for the calculations were derived from the transient tests. After computing the PI parameters, both controllers were applied to both twins. Their motors' behaviors were compared by applying closed-loop step responses to follow a $4 \mathrm{rad} / \mathrm{s}$ reference. The comparison between the left and right motors of the twins can be seen in Figures 10 and 11.

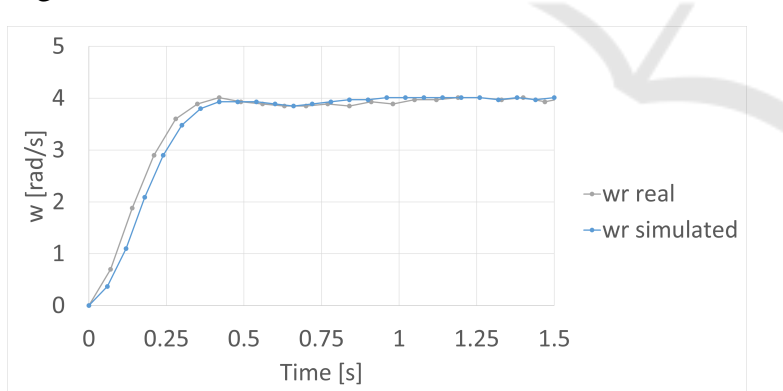

Figure 10: Closed loop response of the right motors of both robots for a $4 \mathrm{rad} / \mathrm{s}$ reference.

As can be seen in the illustrations, each motor and its respective simulated model behave similarly with a time response of approximately $200 \mathrm{~ms}$. Their responses follow the reference with minimum steadystate error and without oscillations during the whole process.

\subsection{Digital Twin}

A software was developed to monitor and provide real-time feedback of both robot's linear and angular

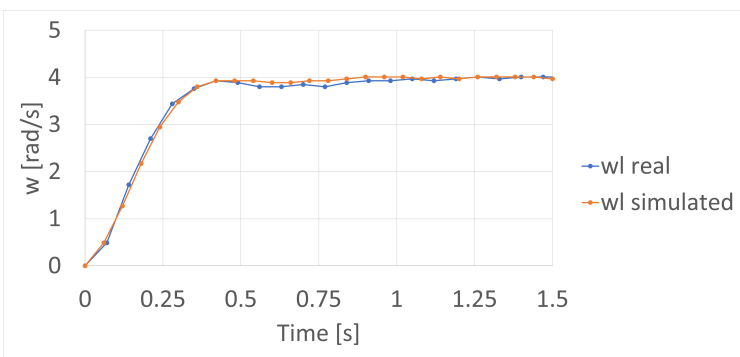

Figure 11: Closed loop response of the left motors of both robots for a $4 \mathrm{rad} / \mathrm{s}$ reference.

speeds. Thus, with the competition simulation scene, it is possible to see the simulated robot and the real robot performing the competition's required tasks simultaneously. The software layout can be seen in Figure 12.

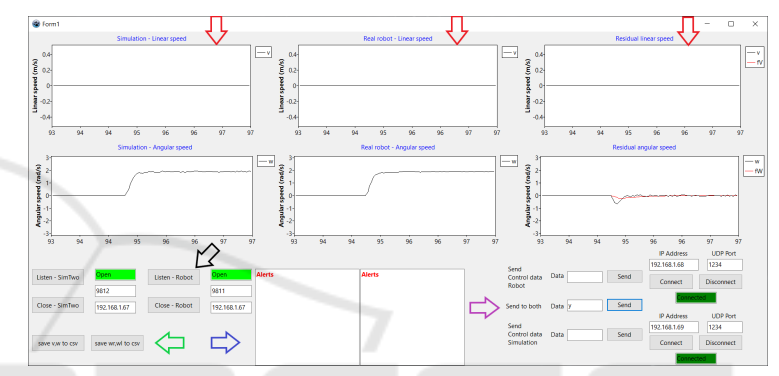

Figure 12: Digital twin software layout.

The charts, displayed by the red arrows in the figure, are dynamic charts that receive the robot's speeds through UDP communication protocol. They are organized in columns, with the first row representing the linear speeds and the second row, the angular speeds. The first column is data received by the simulated robot. The second column, received by the real robot. Finally, the last column represents the respective residual speeds between the twins. Regarding the UDP communication configuration, the receiving end is configured in the location shown by the black arrow. The configuration is made by simply choosing the IP and UDP port that the software will "listen to" to receive data from the twins. The sending end, on the other hand, is configured where the purple arrow is pointing at. In this location, it is possible to send specific commands to each twin or both at the same time. Finally, the software alerts whenever the twins' data diverge by more than a threshold and displays how much time the divergence endured. The alerts show in the white boxes displayed by the blue arrow. The left box shows linear speeds' divergences and the right one, angular speeds' divergences. It is important to emphasize that only the robot's speeds are monitored (robot's centroid reference frame), not the individual motors' speeds. To avoid giving false alerts, 


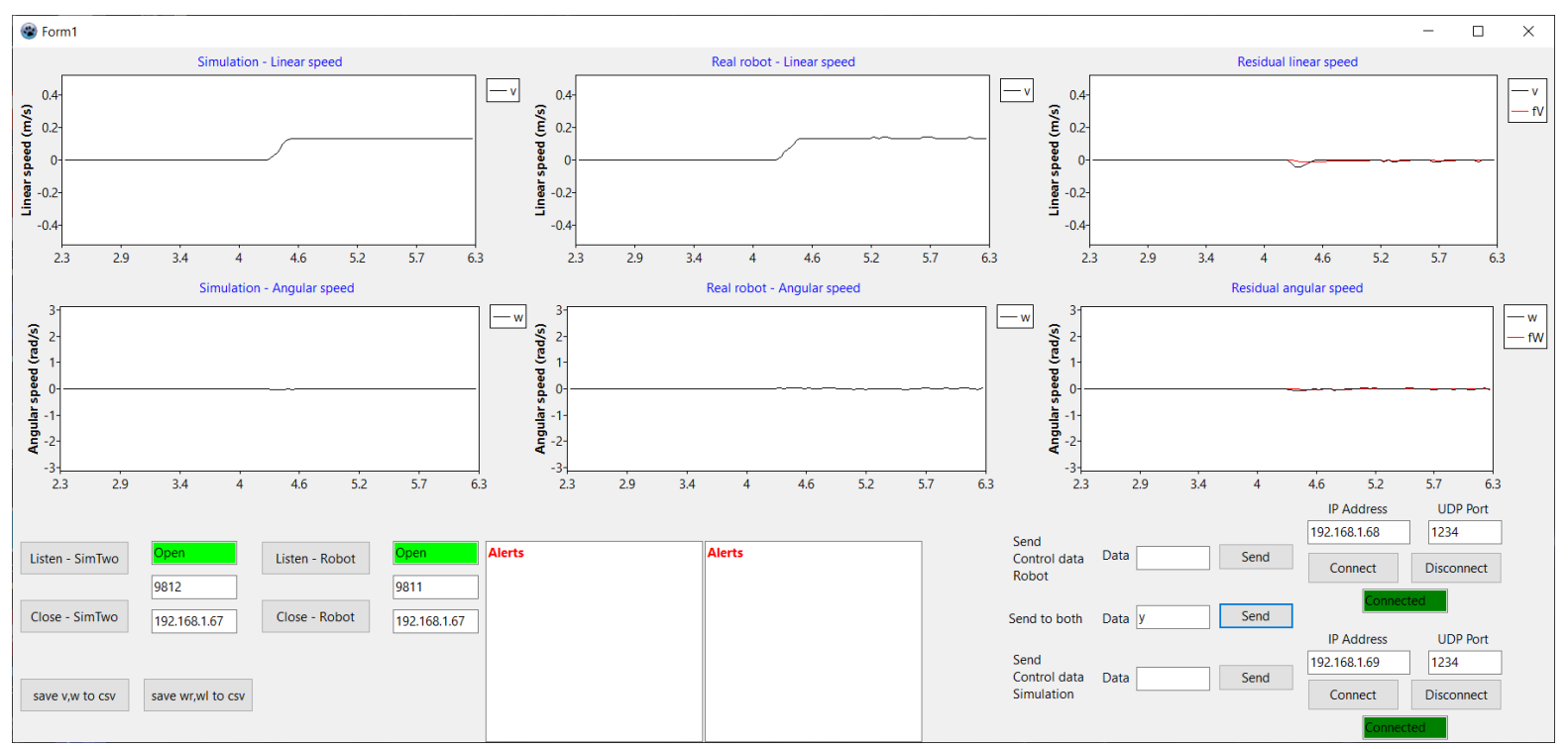

Figure 13: Real robot and digital twin response to a move straight command.

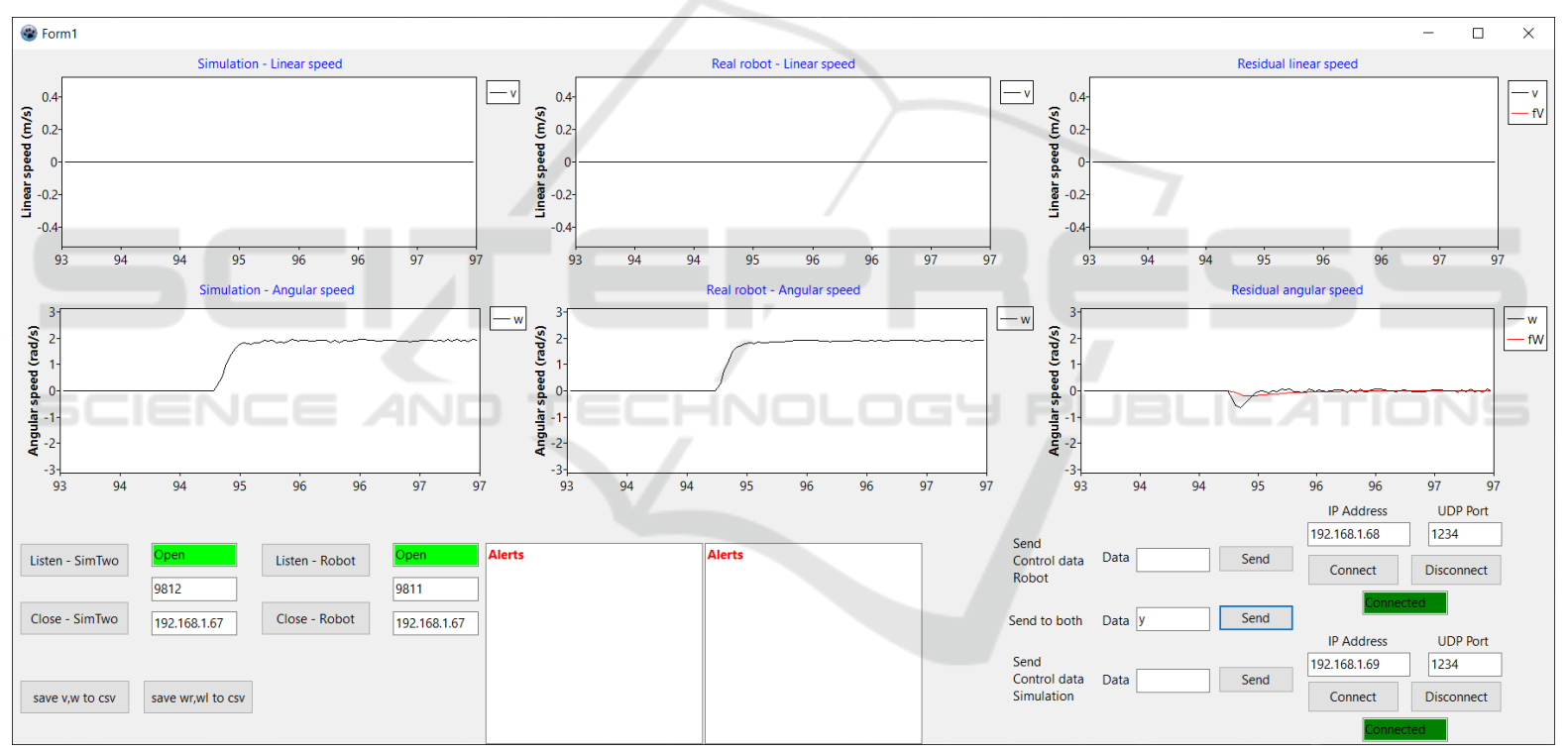

Figure 14: Real robot and digital twin response to a rotate over its own axis command.

influenced by the intrinsic noise present in both twins and by the transient periods of the motors, the residual data is filtered with a first-order IIR filter.

\section{RESULTS}

Three tests were performed to test the digital twin. The idea behind these tests is to give the same command to both twins and analyze if they are operating similarly. The $x$-axis values in the following charts are displayed in seconds with one decimal point in all charts. This was done to not pollute the image. The first test was to command the twins' motors to follow $4 \mathrm{rad} / \mathrm{s}$ angular speed references. The twins' response can be seen in Figure 13.

As can be seen in the illustration, both robots perform similarly. The robot's angular speeds remained 0 , as expected. There is a little disturbance in the residual linear speed, which is foreseen during the transient period. As mentioned before, there are no alerts for two reasons, the first is that only the filtered residual is analyzed. The other is that the software must detect several times the divergence passing a threshold before alerting that it is something wrong. During this period, if the residual values go below the 


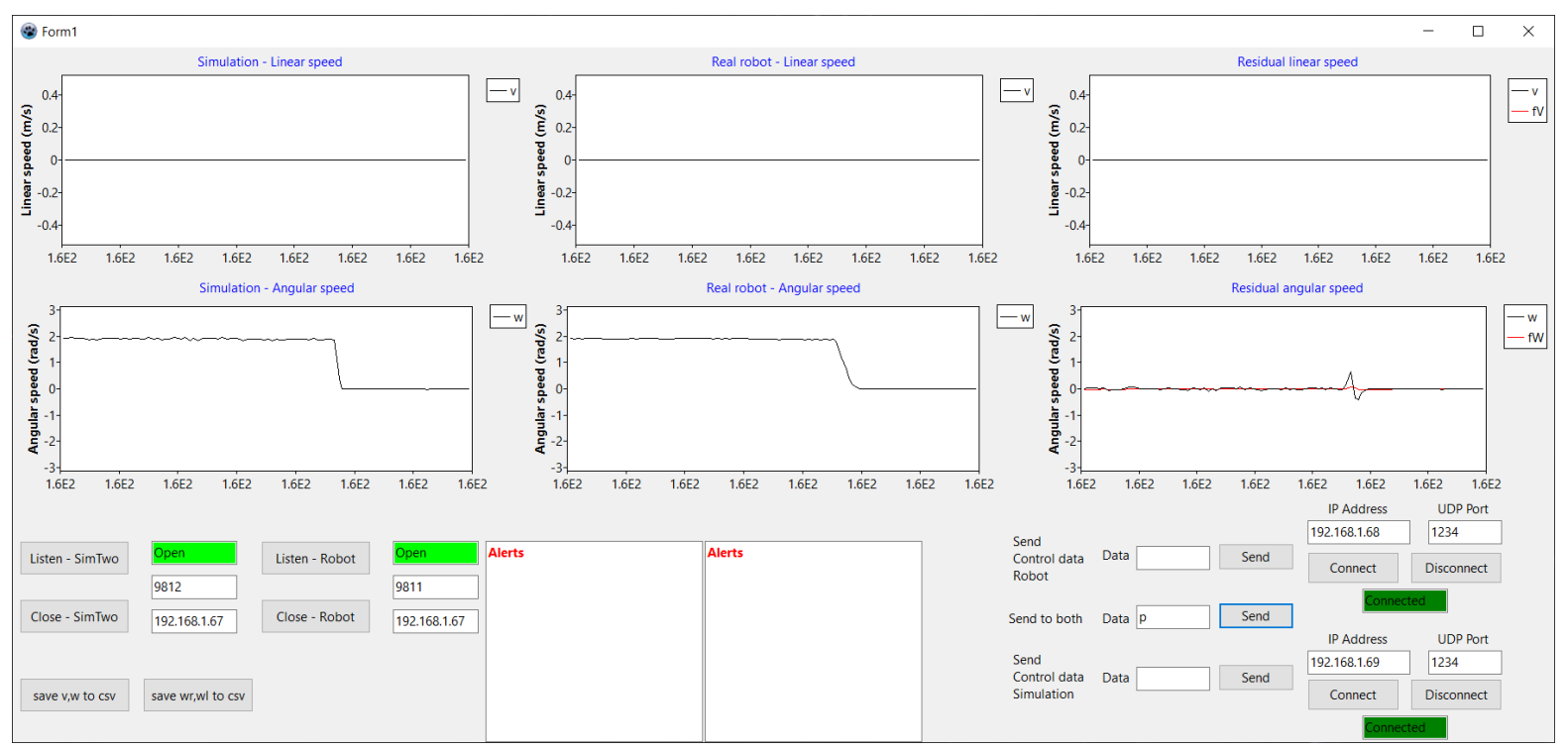

Figure 15: Real robot and digital twin response to a sudden stoppage.

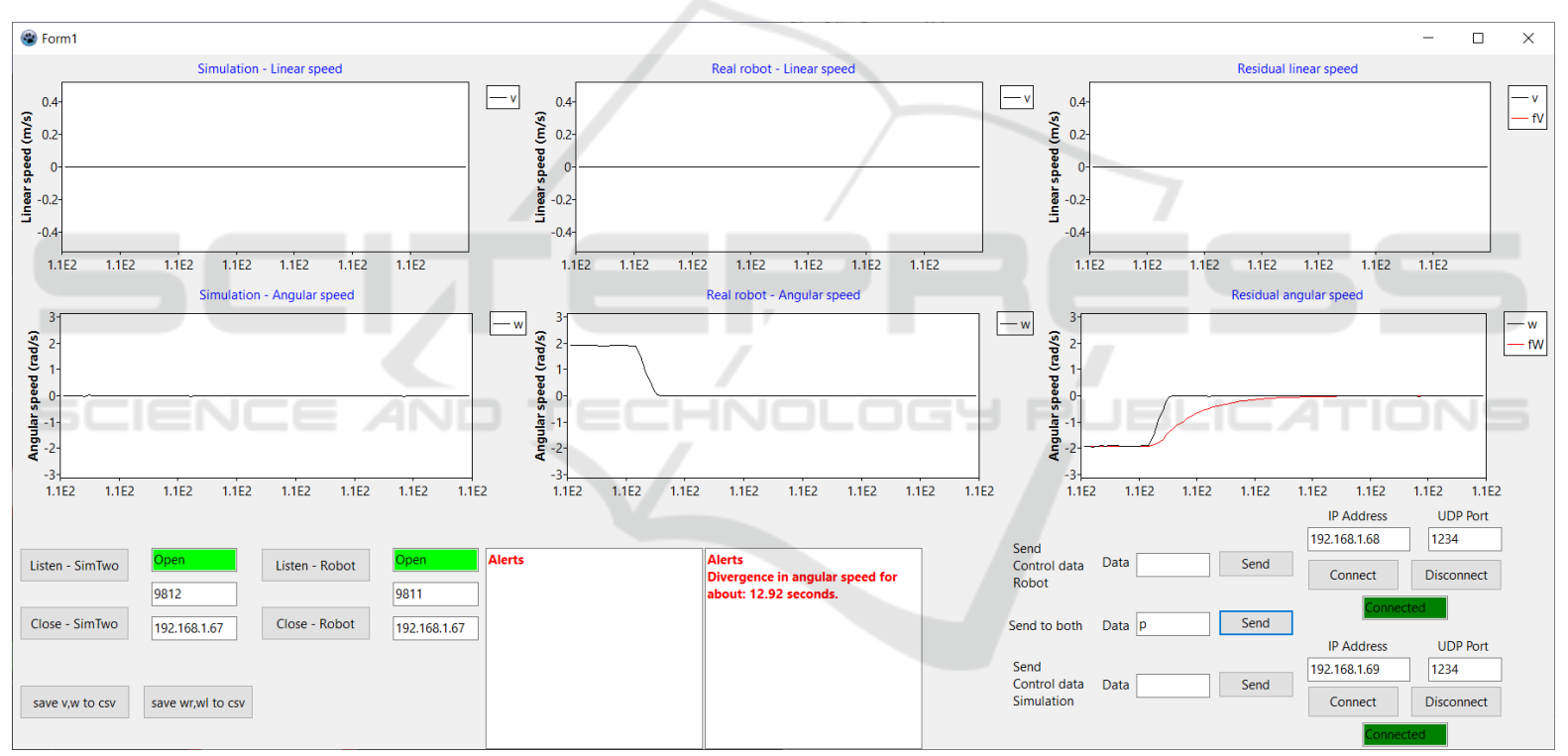

Figure 16: Forced alert by stopping the simulated robot in a rotational test.

threshold, the detection counter is set to 0 .

The second test was to rotate them over their rotational axes by ordering one motor to follow a $4 \mathrm{rad} / \mathrm{s}$ angular speed reference and the other a $-4 \mathrm{rad} / \mathrm{s}$ reference. The robot's behaviors can be seen in Figure 14.

This illustration shows that the robots similarly performed the command, same as the previous test. Their linear speeds remained 0 which is expected since the robots did not move in any direction. Again, the filtered residual damps the transient response and the noise, preventing alerts. Figure 15 shows the robot's response to a sudden stoppage after the rota- tional command.

As can be seen in the figure, there is a peak during the stoppage. This happens because the robot's sudden stoppages, although happened in a very small period, are different. The factors mentioned in 4.1.2, communications in the entire digital twin system, simulator, and different processing hardware could all possibly influence this behavior. Note that this whole process happened very fast as can be seen that the entire charts are in the same second.

The third test was to force an alert by purposely stopping the simulated robot. The test can be seen in Figure 16. 
In this test, the rotational command was given to the twins. However, the motors' voltage in the simulator was purposefully set to 0 , forcing the robot to not rotate. After giving the command to both robots to stop, the alert appeared with the divergence's duration. This behavior is logical because when the robot's stopped, the divergence returned to roughly 0 , below the threshold.

\section{CONCLUSIONS}

This paper presented the development of a digital twin for an AGV for the Robot@Factory Lite competition. The robot's motors were modeled and its parameters were estimated. PI controllers for speed were designed for each motor of the real robot. Applying the same controllers in the simulated robot, the twins behaved similarly. Also, a digital twin software was presented that monitors, in real-time, the behavior of the twins' linear and angular speeds, alerting when they aren't performing similarly for some reason. The divergence detection was designed to be robust to noise and transient periods, preventing false alerts. Finally, three tests were performed, on both twins simultaneously, to show the digital twin performance compared to the real twin. The tests were sufficient to show that the digital twin is performing very similarly, with a negligible difference during transient periods, even with the assumptions made for simplification during the modeling. Therefore, the digital twin will prove useful for the next competitions as well as by the community for other scenarios, with the instant feedback of the robot's performance and by greatly improving the transition to the real scenario.

\section{ACKNOWLEDGEMENTS}

The project that gave rise to these results received the support of a fellowship from "la Caixa" Foundation (ID 100010434). The fellowship code is $\mathrm{LCF} / \mathrm{BQ} / \mathrm{DI} 20 / 11780028$. This work is financed by National Funds through the Portuguese funding agency, FCT - Fundação para a Ciência e a Tecnologia within project UIDB/50014/2020.

\section{REFERENCES}

Lima, J., Costa, P., Brito, T., and Piardi, L. (2019). Hardware-in-the-loop simulation approach for the robot at factory lite competition proposal. In 2019 IEEE International Conference on Autonomous Robot
Systems and Competitions (ICARSC), pages 1-6. IEEE.

P33a (2020). Robot at factory lite. https://github.com/ P33a/RobotAtFactoryLite. Online; accessed 26February-2020.

Pairet, E., Ardón, P., Liu, X., Lopes, J., Hastie, H., and Lohan, K. S. (2019). A digital twin for human-robot interaction. In 2019 14th ACM/IEEE International Conference on Human-Robot Interaction (HRI), pages 372-372.

Paulo, C., José, G., José, L., and Paulo, M. (2011). Simtwo realistic simulator: A tool for the development and validation of robot software. Theory and Applications of Mathematics \& Computer Science, 1(1):17-33.

Rivera, D. E., Morari, M., and Skogestad, S. (1986). Internal model control: Pid controller design. Industrial $\&$ engineering chemistry process design and development, 25(1):252-265.

Smith, R. et al. (2005). Open dynamics engine.

Tao, F., Zhang, H., Liu, A., and Nee, A. Y. C. (2019). Digital twin in industry: State-of-the-art. IEEE Transactions on Industrial Informatics, 15(4):2405-2415.

Tao, F., Zhang, M., Liu, Y., and Nee, A. (2018). Digital twin driven prognostics and health management for complex equipment. Cirp Annals, 67(1):169-172.

Weyer, S., Meyer, T., Ohmer, M., Gorecky, D., and Zühlke, D. (2016). Future modeling and simulation of cps-based factories: an example from the automotive industry. Ifac-Papersonline, 49(31):97-102.

Zhang, H., Liu, Q., Chen, X., Zhang, D., and Leng, J. (2017). A digital twin-based approach for designing and multi-objective optimization of hollow glass production line. IEEE Access, 5:26901-26911. 